\title{
ARX System Identification in Biomedical Applications
}

\author{
Aws Zuhair Sameen, Rosmina Jaafar, Mohammed Hasan Alwan
}

\begin{abstract}
System identification approach is a data driven establishing mathematical model of the system that has been widely applied to the astronomy, automatic control, spaceflight, aviation, economics as well as marine ecology and society. At present, system identification has been widely used in the field of biomedical engineering. The status of system identification technique becomes increasingly important with the rapid development of science and technology in various disciplines. This paper is firstly introduced both linear and nonlinear system identification, then briefly explained the Autoregressive Exogenous (ARX) approach, and finally the applications based on ARX system identification in the biomedical engineering field have been presented.
\end{abstract}

Index Terms-System Identification, Autoregressive Exogenous (ARX), Biomedical Engineering, Healthcare, Biomedical Applications

\section{INTRODUCTION}

System identification defined as the system that is building its mathematical model using the input and output data. The system's behavior is determined by a set of process where each process determines by the chemical and physical properties. System identification has been used in various productions and life applications. The modern SI is very complex, and the internal mechanism is unclear [1]. To establish the mathematical model of the system; designing a rational SI method plays an important role in the process.

Many researchers worked on developing the system identification and each one gave a different definition of identification. In 1962, Zadeh thought in order to determine an equivalent model, the identification depends on the input and output data set from a group of given models [2]. In 1974, Eykhoff defined identified as the problem can be summed up as a kind of calculus, which represents the essential characteristics of the objective system by a model and the understanding of the objective system can be expressed as useful forms by using this model [3]. In 1978, Ljung presents a more specific definition of system identification by saying that data, model and standards are the three elements that are creating the identification and that identification is in accordance with a criterion. The model will be selected depending on the best data fitting from a set of models [4]. A new definition of system identification has been given by Ding [5], which is:

Revised Manuscript Received on December 30, 2019.

Aws Zuhair Sameen, Center of Advanced Electronic and Communication Engineering, Faculty of Engineering and Built Environment, University Kebangsaan Malaysia (UKM), (Email: aws.a.zuhair@ieee.org)

Rosmina Jaafar, Center of Advanced Electronic and Communication Engineering, Faculty of Engineering and Built Environment, University Kebangsaan Malaysia (UKM), (Email:rosmina@ukm.edu.my)

Mohammed Hasan Alwan, Department of Communication, Faculty of Electrical and Electronic, University Tun Hussein Onn Malaysia, Batu Pahat, Johor, Malaysia. (Email:mohammed.h.alrubaye@ ieee.org)
- Designing an appropriate input signal

- Using the experimental input and output data

- $\quad$ Selecting a model class

- Constructing an error criterion function and determining the fitting model by optimizing method

In such cases the process starts by measuring the system behavior and the input data of the system, then without going into the details of what is going on inside, the system tries to determine a mathematical relationship between the input and output where this approach is called system identification.

Initially, system identification has been used with discrete time domain systems. In the last decade, it has been used for continuous time domain analysis [6]. Based on the problem characteristics, the system identification can be classified into three categories which are input class, models' class and criterion.

Depending on Ding's definition, there are three modeling techniques which are white, gray and black boxes where the white box or morphological modelling can be built easily by doing simple experiments to inquire into the involved chemical or physical laws. In other situations when the models are more complex or even impossible to obtain the results in reasonable time due to the nature of the system and processes.

A Gray box model which is also known as semi physical modeling were part of what is going inside the system is known. In this model the system is constructed based on both experimental data and insight into the system. However, the system still has some unknown parameters that can be estimated using system identification.

The black box model builds without having any knowledge of the system and it works based on data driven only. The results of this model describe the dependency of the output on the input data, not how actually the system is working which means the results interpretation are not straightforward. Most system identification algorithms have considered a part of this type. If the model form is known, the estimation of the parameters is easy but in real life situations this is not the case where the model's structure is very complex [7].

Recently, some new system identification methods are constantly emerging and became more able to obtain better identification according to the innovation and the constant development of system identification techniques. The reliability and availability of system identification design techniques expanded beyond the industrial application scope. Biology, medicine, biomedical engineering, psychology, economy, and the environment are examples of

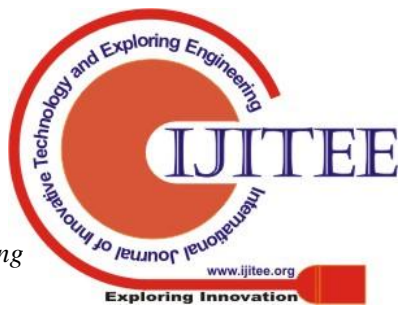




\section{ARX System Identification in Biomedical Applications}

the fields that system identification applied in $[8,9]$.

The rest of this paper will be organized as follow: section II is presenting the types of system identification. Section III is a brief explanation of ARX system identification approach, section IV review of ARX model applications in the field of biomedical engineering, and finally the section $\mathrm{V}$ is the conclusion of the paper.

\section{TYPES OF SYSTEM IDENTIFICATION}

There are two types of system identification which are linear and nonlinear systems. The linear system identification methods work based on determining the error criterion function and the framework of the system where the nonlinear system identification methods are more complex and works with the fields that linear system identification methods are inapplicable [10]. The model parameters of the system are determined according to the input and output data by minimizing the error criterion function.

\section{A. Linear System identification}

According to the input and output data of the system and based on the system's model framework determination as well as the error criterion function, the parameters of the model are determined by minimizing the error function [11]. The traditional methods of identification include least mean square (LMS), maximum likelihood, gradient correction and Autoregressive exogenous (ARX) approach. These approaches are applied to the systems that have linear relationship between the input and the output data. Most of the linear system's properties such as impulse response, stability, convolution, duality can be represented by linear difference equation. The effect on an arbitrary signal of the linear system can be obtained by convolution the input signal with the impulse response of the system where the system's output can be obtained by the convolution integral:

$$
y(t)=g(t) \times x(t)=\int_{0}^{t} g(t-\tau) x(\tau) d \tau
$$

Where $y(t)$ is the output of the system, $x(t)$ is the input signal and $g(t)$ is the impulse response of the system that is presenting the characteristics of the system's dynamic behaviour. The system is identified by calculating the impulse response $g(t)$. If $x(t)$ and $g(t)$ are convolved, the system will give optimally fitted $y(t)$ [12]. These methods are suitable for online identification. Usually, the generalized error criterion function is taken by considering the impact of system noise in the identification process. However, these methods or approaches have disadvantages which the input signal must be known and need to be change relatively where in some dynamic systems they are not satisfied with this condition, the linear system identification approaches are able to produce good results for the linear systems only where they are inapplicable for nonlinear systems and finally the parameter and structure identification cannot be synchronized [1].

\section{B. Non-Linear System Identification}

A nonlinear model is the system's structure that is prepared for virtually describing any dynamic system. In recent years, several non-linear system identification methods have been

implemented based on neural network, fuzzy logic, swarm intelligence optimization algorithms, genetic algorithm, multi-innovation identification algorithm, hierarchical identification and auxiliary model identification [13].

The nonlinear structure can be seen as a mapping concatenation from observed data to regression vector as well as from the regressor space to the output space in the nonlinear mapping. Usually, the latter mapping acts as a basis function expansion which is typically formed from one scalar function that is modified in terms of the location and scale.

The basic techniques for parameters estimation in the nonlinear systems structures are the criterion minimization and the two steps procedures. The basis functions can be determined based on the data and then the coordinates of the approximation function determined using the linear least squares steps.

\section{AUTOREGRESSIVE EXOGENOUS (ARX) MODEL}

The system identification approach is the best way for finding the mathematical description of black box systems. Fig. 1 illustrates the general structure of black box system identification with input and output terminals.

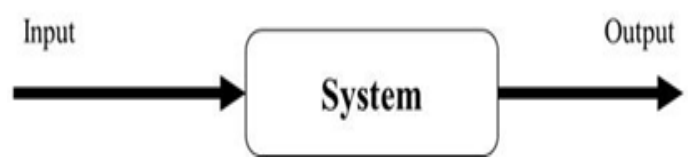

Fig.1 ARX model

If the input and output signals were noise free which is not available in real life, the required measurement number can be determined based on the number of unknown parameters in the system. Mathematical description with finite parameters has been used for real systems model determination. System identification uses statistical methods to implement empirical models through optimal design of a pathway for generating an efficient fitting model for the data [14].

Autoregressive Exogenous (ARX) is one of system identification approaches that is used for continuous time models. It is used to build a series of prediction by estimating the similarity between the input and output signals from the state sequence [15]. The output of the ARX system identification is a linear combination of the input and output signals as well as the prediction error [16]. In general, the main goal of the ARX model is to determine the model's parameters and structure using the input and output data [17]. The ARX system identification standard equation is shown below:

$$
y(t)=\sum_{k=1}^{n} a_{k} y(t-k)+\sum_{k=0}^{m} b_{k} x(t-k)+e(t)
$$


Where $a k$, and $b k$ are unknown parameters that define $g(t)$ which is the impulse response of the system, $n$ and $m$ represent the model order and $e(t)$ is an unobserved residual error [18].

\section{ARX APPLICATIONS IN BIOMEDICAL ENGINEERING\& RESULTS}

\section{A. Pulse Transit Time}

Pulse transit time (PTT) calculation using ARX system identification has been investigated [19]. Two photoplethysmogram (PPG) acquired from the carotid and the toe have been used where the carotid PPG signal act as an input signal and the toe PPG as an output signal to the system. The PTT is calculated based on the impulse response of the system and the fitting between the input and the output signals

[20]. The calculated PTT is used for estimating the blood pressure where the results showed that the calculated PTT using an ARX model is highly correlated to the blood pressure with a mean error of $3.4 \%$. The results are validated by comparing the calculated PTT using System identification to the calculated PTT using the traditional methods of PTT calculation $[6,19,20]$

\section{B. Pulse Wave Velocity}

The ARX system identification method has been used for pulse wave velocity (PWV) calculation which is a good marker of arterial stiffness. PWV can be used for cuffless, continuous and non-invasive BP estimation [21]. PWV is estimated based on calculating the time delay of the ARX system identification by optimum coupling the proximal and distal waveforms based on the impulse response of the system

[18]. The system has been applied on impedance cardiography and peripheral arterial blood pressure waveforms. The calculated PWV is used for estimating the diastolic blood pressure (DBP) where it produces a root mean square error of $5.2 \mathrm{mmHg}$. The results validated by comparing them with the conventional PWV detection methods and the corresponding error were $8.3 \mathrm{mmHg}$.

\section{Central Blood Pressure Estimation}

A novel system for estimating the normalized central blood pressure based on ARX system identification from radial PPG has been investigated [12]. The radial PPG from both right and left wrists have been recorded and used as input and output signals of the system. The results showed that the proposed system is able to produce normalized central blood pressure with a mean fitness of $85.2 \% \pm 2.5$. The results have been validated based on the coefficient of variation which is equal to $3.35 \%$ in the proposed system.

\section{Modeling the Upper Limb Human Vasculature}

Parametric approach based on ARX system identification has been proposed for the upper limb of human vascular tree characterization [22]. The experiment showed that the parametric approach based on the ARX system identification is more powerful compare to the frequency domain approach, especially for short data segments [23]. The photoplethysmogram (PPG) signals non-invasively obtained from the right and left indexes has been used. The results showed that the proposed approach could be efficient for the state of the vascular system monitoring in an independent and relatively from the heart condition. It has been suggested to use this technique for the particular treatment evaluation that is affecting the vascular system where the model coefficients showed a high degree of stability.

\section{E. Monitoring Cardiac Output}

The ARX system identification approach has been used to develop relative cardiac changes estimation technique [24]. The cardiac output estimated by analyzing long time radial arterial blood pressure waveform. The cardiac contractions signal set as the input signal to the system $x(t)$ and radial arterial blood pressure waveform set as the output of the system $y(t)$. The impulse response $g(t)$ of the system is identified which is used to convolve with the input signals to give best fitting to the output in the least square sense.

By assuming constant arterial compliance, the analysis of the technique shows the slow beat to beat changes in the waveform to circumvent the confounding wave reflections. The results of this technique have been validated by comparing the estimated cardiac output to original measurement using invasive swine hemodynamic data. The overall error is reduced by $18 \%$ in the proposed technique based on ARX system identification compared to the original technique.

\section{F. Optimizing Fibromyalgia Treatment}

The ARX system identification approach has been used for developing a dynamical model for assigning naltrexone dosages as a fibromyalgia treatment based on clinical data

[25]. Discrete time modeling using an ARX model with multiple inputs has been used where the clinical data are fitted to a parametric multi-input of the model. The proposed model provides insights a novel design for behavioral health related condition and chronic pain.

\section{G. $\quad$ Stability of Atrial Repolarization Variability}

The relationship between the heart rate and the repolarization variability using ARX system identification based on ECG signals has been investigated to access the bounded input bounded output stability [26]. The process is done by measuring the unstable ECG segments for each subject to quantify the dynamic of the system. The results showed that the proposed method based on ARX system identification produces a better prediction of interval dynamic for both healthy and patient subjects with heart failure.

\section{H. Diabetes Mellitus Control}

ARX system identification has been used together with a predictive functional control algorithm to implement an artificial pancreas for type 1 diabetes mellitus patients [27]. The system identification has been used as a second step to perform the final tuning of the predictive functional control to be ready for implementation. The ARX model is used to isolate the insulin impact of the effect of carbohydrates on the blood glucose variations. 


\section{CONCLUSION}

During the last decade, system identification methods have been applied more and more in the field of biomedical engineering to achieve better results than traditional used methods in this field. ARX is one of the most used approaches of system identification techniques because of its characteristics of producing the results and its linearity properties. ARX system identification has been used mostly with fields that are dealing with vascular system of the human body. The results using ARX system identification are promising and it can be applied in more applications in the field of biomedical engineering.

\section{ACKNOWLEDGMENT}

The authors would like to acknowledge the financial support of the grant from Universiti Kebangsaan Malaysia (GUP-2018-050).

\section{REFERENCES}

1. L. Fu, and P. Li, "The Research Survey of System Identification Method," 5th IEEE International Conference on Intelligent HumanMachine Systems and Cybernetics, 2013.

2. L. Zadeh, "From Circuit Theory to System Theory," Proceedings of the IRE, vol. 50, pp. 856-865, 1962.

3. P. Eykhoff, "System Identification: Parameter and State Estimation," John Wiley \& Sons, 555, 1974.

4. L. Ljung, "Convergence Analysis of Parametric Identification Methods,” IEEE Trans. On Automatic Control, vol. 23, pp. 770-783, 1978.

5. F. Ding, "System Identification, Part A: Introduction to the Identification," Journal of Nanjing University of Information Science and Technology: Natural Science Edition, vol. 3 pp. 1-22, 2011.

6. A. Z. Sameen, R. Jaafar, E. Zahedi and K. B. Gan, "A NovelWaveform Mirroring Technique for Systolic Blood Pressure Estimation from Anacrotic Photoplethysmogram," Journal of Engineering Science and Technology, vol. 13, no. 10, pp. PPP QQQ, 2018.

7. G. P. Rao, and H. Unbehauen, "Identification of Continuous-time Systems," IEE Proceedings-Control Theory Applications, vol. 153, pp. 185-220, 2006.

8. Y. L. Zheng, B. P. Yan, Y. T. Zhang, and C. C. Y. Poon,

"Noninvasive Characterization of Vascular Tone byModelBased System Identification in Healthy and Heart

Failure Patients," Annals of Biomedical Engineering, vol. 43, pp. 2242-2252, 2015.

9. L. Batista, T. Bastogne, E. Djermoune, Identification of Dynamical Biological Systems Based on Random Effects Models," Conf. Proc. IEEE Eng. Med. Biol. Soc., 2015

10. D. Liu, "System Identification Methods and Applications,"Beijing: National Defense Industry Press, 2010.

11. D. Chinarro, "System Identification Techniques," In book:System Engineering Applied to Fuenmayor Karst Aquifer (San Julián de Banzo, Huesca) and Collins Glacier (King George Island, Antarctica), Springer Theses, pp. 11-51, 2014.

12. E. Zahedi, V. Sohani, M. A. M. Ali, K. Chellappan, K. and K.B. Gan, "Experimental Feasibility Study of Estimation of theNormalized Central Blood Pressure Waveform from RadialPhotoplethysmogram," Journal of Healthcare Engineering, vol.6, no. 1, pp. 121-144, 2015.

13. J. Sjöberg, Q. Zhang, L. Ljung, A. Benveniste, B. Delyon, P. Y. Glorennec, H. Hjalmarsson, and A. Juditsky, "NonlinearBlackbox Modeling in System Identification: A UnifiedOverview," Automatica, vol. 31, pp. 1691-1724, 1995.

14. H. Karimipour, H. T. Shandiz, and E. Zahedi, "DiabeticDiagnose Test Based on PPG Signal and IdentificationSystem," Journal of Biomedical Science and Engineering, vol.2, pp. 465-469, 2009.

15. M. Jansson, "Subspace identification and ARX modeling,"Royal Institution of Technology, Stockholm, Sweden, 2003.

16. P. Giassi, S. Okida, M. G. Oliveira, and R. Moraes, "Validation of the inverse pulse wave transit time series as surrogate of systolic blood pressure in MVAR modeling," IEEETransactions on Biomedical Engineering, vol. 60, no. 11, pp. 3176-3184, 2013.
17. S. Rachad, H. Fouraiji, B. Bensassi, "Identification Approach for a Production System using ARX Model," International Conference on Logistics Operations Management, 2014.

18. D. Xu, K. L. Ryan, C. A. Richards, G. Zhang, V. A.Convertino, and R. Mukkamala, "Improved Pulse Transit TimeEstimation by System Identification Analysis of Proximal and Distal Waveforms," American Journal of Psychology, Heart and Circulatory Physiology, vol. 301, no. 4, pp. H1389-H1395, 2011.

19. A. Z. Sameen, and E. Zahedi, "Time Delay Estimation between Two Biosignals using System Identification," IEEEInternational Conference on Smart Sensors and Application (ICSSA), Kuala Lumpur, Malaysia, 2015

20. A. Z. Sameen, R. Jaafar, M. A. M. Yahya, "Pulse Transit TimeEstimation from Anacrotic Photoplethysmography Waveforms," IEEE International Conference on Robotics, Automation and Sciences (ICORAS), Melaka, Malaysia, 2017.

21. D. $\mathrm{Xu}, \mathrm{K} . \mathrm{L}$. Ryan, C. A. Rickards, G. Zhang, V. A.Convertino, and R. Mukkamala, "Robust Pulse Wave Velocity Estimation by Application of System Identification to Proximal and Distal Arterial Waveforms," $32^{\text {nd }}$ Annual International Conference of the IEEE EMBS, Buenos Aires, Argentina, 2010.

22. E. Zahedi, and M. A. M. Ali, "Parametric DifferentialApproach for Modeling the Upper Limb Human Vasculature," Proceedings of the 26th Annual International Conference of the IEEE EMBS, San Francisco, CA, USA, $1^{\text {st }}-5^{\text {th }}$ September 2004

23. M. H. Sherebrin, R. Z. Sherebrin, "Frequency Analysis of ThePeripheral Pulse Wave Detected in the Finger with A Photoplethysmograph,” IEEE Trans. Biomed. Eng., vol. 37, no. 3, pp. 313-317, March 1990.

24. M. Moslehpour, G. Zhang, and R. Mukkamala, "CardiacOutput Monitoring by Long Time Interval Analysis of a Radial Arterial Blood Pressure Waveform with Correction for ArterialCompliance Changes using Pulse Transit Time," $33^{\text {rd }}$ Annual International Conference of the IEEE EMBS, Boston, Massachusetts USA, 2011.

25. S. Deshpande, N. N. Nandola, D. E. Rivera, and J. W. Younger, "Optimized Treatment of Fibromyalgia using System Identification and Hybrid Model Predictive Control," Control Engineering Practice, vol. 33, pp. 161-173, 2014

26. J. Sivaramana, G. Umab, P. Langleyc, M. Umapathyb, S.Venkatesand, and G. Palanikumar, "A Study on StabilityAnalysis of Atrial Repolarization Variability using ARXModel in Sinus Rhythm and Atrial Tachycardia ECGs," Computer Methods and Programs in Biomedicine, 2016.

27. L. Griva, M. Breton, D. Chernavvsky, and M. Basualdo,"Commissioning Procedure for Predictive Control Based onARX Models of Type 1 Diabetes Mellitus Patients," IFAC Papers Online, vol. 50, pp. 11023-11028, 2017. 